\section{Emergency medicine in differently resourced settings: what can we offer each other?}

\section{E Molyneux, A Robertson}

\section{The provision of emergency medicine in Malawi is described and compared with that found in countries with more resources.}

E mergencies are as old as humanity. Specialised emergency care is relatively new. Emergency medicine in well resourced parts of the world has become increasingly complex, coordinated, and slick. Expectations of good practice from the public and from inpatient hospital colleagues are high. Experienced and specialised nursing and medical staff run excellent services with high patient turnover. Professional organisations representing emergency medicine make recommendations to government about staffing, training requirements, and back up services.

But what of the universal picture? In most resource poor areas of the world it is very different. There are no prehospital general practice services and no regular ambulance services. Health centres provide primary care. These are staffed by one "clinical officer" (a paramedic trained in diagnosis and treatment) and one or two nurses who have available a few basic drugs. Self referred and health centre referred patients attend district or central hospitals. In these hospitals trauma cases are seen in a side room (called "casualty") of the outpatients department. A clinical officer (CO) or medical assistant (MA) attends. Nominal supervision is given by the district medical officer, or in central hospitals by the orthopaedic or general surgical department. All other (medical) emergencies line up to see a CO or MA in outpatients. The queues are long and the staff are few. Laboratory investigations are minimal (perhaps a peripheral blood film for malaria parasites, a haemoglobin concentration, urine and stool microscopy) The World Health Organisation reports that the average patient waits 60 to 120 minutes for a 54 seconds interview with a CO. ${ }^{1}$ This may be hard on the patients but it is also hard on the clinician. It is not easy to spend 54 seconds with each of 250 patients every day for six days a week.

Numbers are highest for paediatric emergencies. In district hospitals the standard outpatients area is designed in such a way that antenatal care, the giving of immunisation, and treating of sick children are done together. In larger hospitals efforts have been made to bring some order to the management of emergencies. Outpatients and emergencies are still muddled together but some triage is undertaken so that rehydrated children are treated in an oral dehydration room or corner and admissions sent to the inpatient wards. But the outpatient departments are usually run separately from the inpatient wards and staff in each section seldom meet. Communication is poor. Bleeps are not usually available, telephones are few and not well placed to call for urgent help.

In the Queen Elizabeth Central Hospital, Blantyre, Malawi, we have tried, and in part succeeded, in introducing a paediatric emergency care service. This has been possible in a new purpose built department with extra nurses (funded by charitable donations) and leadership from a small number of people intent on its success. Staff have been trained in triage, resuscitation, trauma, and the initial management of medical emergencies. Inpatient paediatric medical staff admit from the unit and are present throughout the day. A short stay ward allows for observation over a six hour period. The paediatric emergency unit is very busy; in the malaria season up to 135 children are admitted and 20 or more observed daily in the short stay ward. Attendances to this accident and emergency unit can reach 800 per day. It demands commitment, skill, and hard work to get through each day. But since the department opened its doors to patients, the inpatient case fatality rate, which used to be $10 \%-15 \%$, has been reduced to $5 \%-8 \%$

\section{PROBLEMS OF DELIVERING EMERGENCY CARE IN RESOURCE POOR SETTINGS \\ Staffing}

Employing and retaining good staff is a universal problem and in Africa, where it is not unusual for one clinician to treat 200 patients a day, assisted by one or two nurses, even the most committed staff become demoralised by chronic overwork. A senior trained clinician needs to be based in the department to supervise clinical care, and to manage the unit.

\section{Training}

Accident and emergency medicine is not recognised as a specialty in most resource strained countries. The team in an accident and emergency unit needs training, supervision, and leadership. Specialists need to be trained who will not only give clinical direction but will lobby for their cause.

\section{Work environment}

Consumables-drugs, cannulas, strapping, plaster of Paris are frequently scarce. Maintenance of equipment such as oxygen concentrators is a recurrent problem because of lack of spare parts or expertise. The voltage of the electricity supply fluctuates and damages sensitive electronic instruments. Basic cleanliness is a major problem. The huge volume of patients, some unaccustomed to flush toilets puts a strain on the ability to keep a unit clean. Add mud in the rainy season and it becomes impossible.

The positive side of emergency care There is a wealth of clinical medicine. Trainees get vast experience in a wide range of emergencies. Interns see more and do more hands on care in three months than most paediatric accident and emergency trainees and hospital paediatric trainees will see and do in UK in two years. Medical staff have to use their clinical and diagnostic skills, develop responsibility, learn to be adaptable and to think laterally. It is stressful, challenging, and often deeply rewarding.

\section{HOW CAN EMERGENCY UNITS IN DIFFERENTLY RESOURCED PARTS OF THE WORLD HELP EACH OTHER?}

The practice of emergency care is very different in well resourced and poorly resourced settings but each has something to offer the other.

Professional emergency medicine organisations could be an advocate for emergency medicine in less advantaged parts of the world. It could help individual countries to develop courses in life support and special training in emergency care.

Resource rich units could second doctors and/or nurses to work for three to four months in a tropical setting. Trainees and senior staff could help in teaching, in organisation, and in clinical care in the receiving department. Resource rich units could receive junior doctors from less fortunate departments into their units for clinical attachments or to take up substantive posts. Resource strained units would welcome the ideas, 
enthusiasm, interest, and practical help of staff of well resourced units. Perhaps two such hospitals could be twinned. Visitors to a poorer unit would also benefit. They would hone up on clinical and diagnostic skills. They would enjoy a different culture and be humbled by people's gratitude for care. It would be a time to concentrate on clinical medicine and teaching and problems outside the NHS As one visiting trainee to our department put it: "It reminded me of why I went into medicine in the first place".

Emerg Med J 2002;19:378-379

\section{Authors' affiliations}

E Molyneux, A Robertson, College of Medicine, Box 360, Blantyre, Malawi, C Africa

Correspondence to: Dr E Molyneux emolyneux@malawi.net
REFERENCE

1 WHO. Health is a precious asset. Geneva: WHO/RSD/00.1, 2000

AN ADDENDUM WRITTEN BY JIM WARDROPE, EDITOR, EMERGENCY MEDICINE JOURNAL TO STIMULATE DEBATE

Could the EMJ do more for under resourced countries? Free online provision of the $E M J$ and other $B M J$ group journals to about 100 countries is welcome. However, we need to see much more content that is of relevance to those practising emergency care in Africa, South America, and the Indian subcontinent. There are many ways that clinicians practising in these countries can provide material that would be of great benefit to all. We need to expand our "evidence based practice" although this will always be tempered by economic reality. We would be interested by a wider "Journal scan" to include some articles on tropical diseases.

If the $E M J$ really wants to be relevant to us then it must change, a change that I think would make it a better and more interesting read for all clinicians. Equally those practising in the developing world need to be proactive, learn how we can submit copy to the journal, and seek help and assistance where needed.

Everyone pays lip service to the ideals of closing the gap between the health care provision in the "west" and the "south". However, the gap seems to be widening, as clinicians we should try to do something to narrow this divide. 